\title{
Specialized Respiratory Management for Acute Cervical Spinal Cord Injury: A Retrospective Analysis
}

\author{
Sandra Lynn Wong, BA, RCP, ${ }^{1}$ Kazuko Shem, $M D,{ }^{2}$ and James Crew, $M D^{2}$ \\ ${ }^{1}$ Department of Respiratory Care Services, ${ }^{2}$ Department of Physical Medicine and Rehabilitation, \\ Santa Clara Valley Medical Center, San Jose, California
}

\begin{abstract}
Background: In individuals with cervical spinal cord injury (SCI), respiratory complications arise within hours to days of injury. Paralysis of the respiratory muscles predisposes the patient toward respiratory failure. Respiratory complications after cervical SCI include hypoventilation, hypercapnea, reduction in surfactant production, mucus plugging, atelectasis, and pneumonia. Ultimately, the patient must use increased work to breathe, which results in respiratory fatigue and may eventually require intubation for mechanical ventilation. Without specialized respiratory management for individuals with tetraplegia, recurrent pneumonias, bronchoscopies, and difficulty in maintaining a stable respiratory status will persist. Objective: This retrospective analysis examined the effectiveness of specialized respiratory management utilized in a regional SCI center. Methods: Individuals with $\mathrm{Cl}-\mathrm{C} 4 \mathrm{SCl}(\mathrm{N}=24)$ were the focus of this study as these neurological levels present with the most complicated respiratory status. Results: All of the study patients' respiratory status improved with the specialized respiratory management administered in the $\mathrm{SCl}$ specialty unit. For a majority of these patients, respiratory improvements were noted within 1 week of admission to our $\mathrm{SCl}$ unit. Conclusion: Utilization of high tidal volume ventilation, high frequency percussive ventilation, and mechanical insufflationexsufflation have demonstrated efficacy in stabilizing the respiratory status of these individuals. Optimizing respiratory status enables the patients to participate in rehabilitation therapies, allows for the opportunity to vocalize, and results in fewer days on mechanical ventilation for patients who are weanable. Key words: atelectasis, mechanical ventilation, pneumonia, respiratory complications, respiratory therapy, spinal cord injury, tetraplegia
\end{abstract}

$\mathrm{R}$ espiratory complications are the leading cause of morbidity in individuals with cervical spinal cord injury (SCI), therefore specialized respiratory management is critical after injury. In the acute hospitalization phase, respiratory complications are prevalent, affecting $84 \%$ of persons with $\mathrm{C} 1$ to $\mathrm{C} 4 \mathrm{SCI}{ }^{1}{ }^{\text {Atelectasis }}$ $(36.4 \%)$, pneumonia $(31.4 \%)$, and ventilatory failure $(22.6 \%)$ are the most common respiratory issues for this group during the acute phase. ${ }^{1}$ There is a direct correlation between the level of injury diagnosed between the American Spinal Injury Association (ASIA) Impairment Scale (AIS) and the development of respiratory complications. In individuals with SCI who have neurological levels of $\mathrm{C} 3$ and caudal who are able to spontaneously breathe initially, ventilator failure can occur 4.5 \pm 1.2 days after injury and last for an average of 5 weeks. ${ }^{1}$

Individuals with an AIS A SCI and those with high cervical injuries are at higher risk for respiratory complications. ${ }^{2}$ Specialized interventions utilized by respiratory care practitioners in dedicated SCI units have been shown to effectively improve the respiratory status of patients transferred from facilities that do not specialize in SCI care. ${ }^{3,4}$ Secretion and ventilation management of individuals with acute cervical SCI differs greatly from that required by individuals with pulmonary dysfunction secondary to nonneurological injuries. ${ }^{3,5}$ Individuals without neurological injuries have an intact cough that is critical for secretion mobilization and expulsion, and surfactant production is present. The lungs in individuals with acute complete cervical SCI have decreased lung expansion, a highly impaired cough due to the weakness of expiratory muscles, impairment of the elastic recoil of the diaphragm and lung, increased secretion production due to an unopposed parasympathetic stimulation, and decreased surfactant production. ${ }^{6,7}$ Therefore, interventions to aid secretion mobilization are crucial in preventing mucus plugging, atelectasis,

Top Spinal Cord Inj Rehabil 2012;18(4):283-290

(C) 2012 Thomas Land Publishers, Inc.

www.thomasland.com

doi: $10.1310 /$ sci1804-283 
pneumonia, and ventilatory failure following injury. Because the cough flow and effectiveness are greatly reduced with a cervical SCI, the ability to clear secretions is profoundly affected., 8 Aggressive respiratory therapy interventions need to begin immediately after injury, which is further described in detail below. ${ }^{10}$

Facilities not familiar with the respiratory impairments of individuals with cervical SCI may manage these individuals in the same manner as they would an individual with nonneuromuscular injuries. Unfortunately, this may lead to atelectasis, increased secretions, hypercapnea, pneumonias, bronchoscopies, a higher $\mathrm{FiO}_{2}$ requirement, reintubations, inability to vocalize via minimum leak technique or the use of a speaking valve, immobility, and unsuccessful weaning from the ventilator. ${ }^{10}$ For individuals with motor incomplete cervical injuries and lower (C5 and caudal) motor complete SCI, spontaneous respiratory effort may initially be supported by noninvasive bi-level positive airway pressure ventilation (BiPAP), incentive spirometry, positive end-expiratory pressure (PEEP) therapy, vibratory therapy, or lung expansion therapies. BiPAP is a machine that augments the patient's spontaneous breaths, thereby allowing the patient to breathe easier and more deeply with assisted inspiratory positive pressure breaths. ${ }^{11}$ BiPAP may also increase oxygenation by keeping the airways open by applying positive pressure during exhalation. ${ }^{12}$ Both PEEP and vibratory therapy apply resistance on exhalation, enhancing secretion mobilization and treating atelectasis. ${ }^{3}$ Delivering positive airway pressure improves resting lung ventilation by increasing the tidal volume $(\mathrm{Vt})$, prevents and/ or treats atelectasis, improves ventilation due to a better exchange of gases, and aids pulmonary secretion mobilization. Deep breathing and coughing exercises the respiratory muscles and aids in the mobilization and expulsion of pulmonary secretions and should be done by the patient several times during the day. ${ }^{13}$ If these interventions fail to improve the respiratory status, more aggressive specialized respiratory interventions such as those utilized for individuals with high cervical SCI (high tidal volume ventilation, high frequency percussive ventilation, or mechanical insufflation-exsufflation) may need to be implemented.

\section{High Tidal Volume Ventilation (HVtV)}

Ventilating a patient with tidal volumes up to $20 \mathrm{~mL} / \mathrm{kg}$ of ideal body weight (BW) goes against the standard 8 to $10 \mathrm{~mL} / \mathrm{kg}^{14}$ or the ARDSnet protocol of tidal volumes 6 to $8 \mathrm{~mL} / \mathrm{kg}$ of ideal BW ventilation ${ }^{15}$ that is instituted in most facilities that do not specialize in SCI. The fear of barotrauma resulting from high volume ventilation has been described in many articles; but for individuals with complete tetraplegia, high tidal volumes up to 20 $\mathrm{mL} / \mathrm{kg}$ of BW have been beneficial in stabilizing their respiratory ventilation. ${ }^{16-18}$

Peak airway pressures with these higher than normal tidal volumes rarely exceed $30 \mathrm{~cm}$ of water pressure (cwp) due to the flaccid muscle tone of the individuals with acute SCI without acute respiratory distress syndrome (ARDS). Low tidal volumes delivered to individuals with acute tetraplegia can lead to atelectasis, mucous plugging, and decreased production of surfactant. ${ }^{16}$ Poor lung expansion results in inadequate gas exchange of $\mathrm{O}_{2}$ and $\mathrm{CO}_{2}$ leading to atelectasis. Surfactant production is decreased due to the shallow breathing volumes of the individuals with tetraplegia. ${ }^{19}$

High tidal volume ventilation recruits distal airways, stimulates surfactant production, and improves oxygenation. Mechanical ventilation settings with tidal volume starting at $12 \mathrm{~mL} / \mathrm{kg}$ of ideal BW, a respiratory rate of 8 to 10 breaths per minute, and volume control ventilation mode is utilized in our SCI unit. A baseline arterial blood gas and end tidal $\mathrm{CO}_{2}$ are obtained. Further implementations of $\mathrm{HVtV}$ are done by gradually increasing the tidal volume daily by $100 \mathrm{~mL}$ increments monitoring arterial blood gases and/ or end tidal $\mathrm{CO}_{2}$ and monitoring peak airway pressure and plateau pressure with each tidal volume change. ${ }^{10}$ Dead space may need to be added to the ventilator circuit to compensate for the lower $\mathrm{PaCO}_{2}$ values (respiratory alkalosis) that will result from the higher tidal volume being delivered to the patient; the $\mathrm{PaCO}_{2}$ should be targeted within 
the normal range of 35 to $45 \mathrm{~mm} \mathrm{Hg}$. Each 6 in. of dead space equals $50 \mathrm{~mL}$ of volume. Bi-level ventilation (pressure support with PEEP) has not been shown to be effective in treating atelectasis in the individuals with acute high tetraplegia, because due to the loss or impairment of the elastic recoil of the diaphragm, the individuals with high tetraplegia have difficulty in exhaling against any PEEP. ${ }^{10}$ When a newly received patient is being ventilated on any pressure support with PEEP, the patient is switched over to volume ventilation, and the PEEP is slowly weaned to zero as the tidal volume is gradually increased. When the patient with SCI is ventilated on higher tidal volume, atelectasis and pneumonia begin to resolve.

\section{Secretion Management}

The loss of effectively mobilizing pulmonary secretions in individuals with acute tetraplegia quickly leads to impaired oxygenation, respiratory fatigue, and potentially respiratory failure. In addition, secretion retention contributes to atelectasis and pneumonia. Specialized respiratory interventions need to be implemented as soon as possible for secretion management. Intrapulmonary Percussive Ventilation (IPV; Percussionaire Corp., Sandpoint, Idaho) is a combination of high frequency ventilations (up to 300 breaths per minute), high density humidification, therapeutic aerosol delivery, medication nebulizer, and intrapulmonary chest percussion.

High frequency percussive ventilation (HFPV) is used as a treatment modality for individuals with SCI. With the high frequency low pressure percussive ventilations via IPV, a consistent pressure wedge is maintained while a high velocity inflow keeps the airways open and enhances intrabronchial secretion mobilization. By aiding in effective secretion mobilization, HFPV improves alveolar ventilation. The high density aerosol delivered can be as high as $1 \mathrm{cc} / \mathrm{min}$. Along with normal saline, bronchodilators, and/or mucolytics, the adhesive and cohesive properties of retained airway secretions are reduced. The indications for HFPV are for mobilization of retained endobronchial secretions and the resolution of diffuse patchy atelectasis. ${ }^{20} \mathrm{HFPV}$ can be administered in-line with the ventilator circuit, which does not interfere with the patient's conventional mechanical ventilation. ${ }^{21}$

Mechanical insufflation-exsufflation (MIE) is a noninvasive therapy that simulates a cough cycle. It safely and consistently aids in the removal of pulmonary secretions in patients with an ineffective cough (peak cough flow $<270$ $\mathrm{L} / \mathrm{m}) .{ }^{22,23}$ MIE mobilizes secretions by gradually applying a positive pressure to the airways then rapidly shifting to negative pressure. The rapid shift in pressure produces a high expiratory flow simulating a natural cough. One can observe the tracheal secretions being projected up the patient's tracheal tube for easy removal. It can reduce intrapulmonary trauma that may result from repeated tracheal suctioning with a suction catheter. MIE use has been shown to improve pulmonary function parameters and is associated with decreased respiratory hospitalizations for persons with SCI who have a history of smoking. ${ }^{24,25}$ Although there is often a concern for barotrauma with MIE use in those with SCI, pneumothorax is uncommon when MIE is utilized at appropriate pressures such as $+40 \mathrm{~mm} \mathrm{Hg}$ and $-40 \mathrm{~mm} \mathrm{Hg} .{ }^{26}$

At our center, an individual with cervical SCI with atelectasis and/or pneumonia will receive HFPV therapy followed by MIE every 2 to 4 hours around the clock. It is not uncommon for a bedside respiratory therapy session of HFPV treatment with MIE therapy to be 30 to 45 minutes in length, but the use of MIE has been shown to shorten the length of the treatment period by about 17 minutes. ${ }^{27}$ It is an aggressive and diligent regimen, and respiratory improvement is seen within a matter of days.

\section{Purpose}

HVtV, HFPV, and MIE treatments are "standard" respiratory care interventions in our SCI unit for patients presenting with acute high cervical injury and are utilized more frequently for those with atelectasis and/or pneumonia. To examine the efficacy of our regimen, the respiratory status of individuals with acute tetraplegia prior to admission was compared with the respiratory 
status and outcomes of these same patients while being treated in our SCI unit. Ventilator settings, level of injury, AIS impairment class, incidents of pneumonia, number of bronchoscopies performed, date of injury, ventilator weaning attempts, $\mathrm{FiO} 2$ requirements, types of respiratory care management interventions administered, and the patients' respiratory outcomes were compared.

\section{Methods}

Twenty-four individuals with high cervical (C1C4) SCI who were admitted to our SCI specialty unit over the previous 2 years were studied. There were 22 males and 2 females. Average age was 33.4 years (SD 16.6). The ethnicities of the 24 study patients were African American $(n=3)$, Asian $(n=3)$, Hispanic $(n=7)$, and white $(n=11)$. In terms of etiology, 6 injuries were the result of gunshot wounds, 8 were due to motor vehicle accidents, 4 were from falls, 3 were the result of diving accidents, 1 was from a cervicomedullary tumor, 1 was the result of a bicycle accident, and 1 was from a sports accident. Average time from injury to transfer to the SCI specialty unit was 33.8 days (SD 24.4). The distribution of AIS classification was as follows: C1 AIS A ( $n=6)$, C1 AIS D $(n=1)$, C2 AIS A $(n=1)$, C3 AIS A $(n=4)$, C4 AIS A $(n=8)$, C4 AIS B $(n=3)$, and C4 AIS C $(n=1)$. Four patients had a history of smoking. One patient had premorbid history of asthma, diabetes, and cancer. One patient had premorbid history of coronary artery disease and congestive heart failure with a baseline ejection fraction of about $30 \%$. Two patients had impaired diaphragms. Body mass index (BMI) ranged from 20.13 to 35.45 with the average of 25.82 ( $S D$ 16.6).

\section{Results}

The following is a summary of the subjects' respiratory status prior to admission to the SCI specialty unit. All of the patients required oxygen supplementation with an average of $38.5 \% \mathrm{FiO} 2$ ( $S D$ 12.6). One patient's $\mathrm{FiO} 2$ requirement was $90 \%$. Over half of the patients $(n=14 ; 58 \%)$ had bronchoscopies. Twenty-three patients (96\%) had pneumonia. One patient had bronchitis. Only 2 $(8 \%)$ of the study patients were ventilated with tidal volumes greater than $12 \mathrm{~mL} / \mathrm{kg}$ ideal BW. The average ventilator tidal volume of the subjects was $685.5 \mathrm{~mL}$ (SD 173.9). Five patients were ventilated on pressure support with PEEP; the average pressure support was 12.4 (SD 1.8). The average PEEP setting was 5.2 (SD 1.9). Four (80\%) of these patients ventilated on pressure support with PEEP had one or more bronchoscopies performed prior to transfer to the SCI unit. Very few subjects were receiving HFPV $(n=5 ; 10 \%)$ or MIE therapy $(n=1$; $4 \%$ ). Only one subject had tried a speaking valve previously, which had failed. All of the patients failed or did not have ventilator weaning attempts. None of the individuals were reported to have been up in a wheelchair.

Next is a summary of the subjects' respiratory status after treatment in the SCI specialty unit with $\mathrm{HVtV}, \mathrm{HFPV}$, and MIE treatment. Tidal volume of all patients were stabilized at 12 to $15 \mathrm{~mL} / \mathrm{kg}$ ideal BW (average tidal volume $=1037.50 \mathrm{~mL}$; SD 140.8). All patients were treated with MIE therapy, and $22(92 \%)$ of the study patients were treated with HFPV therapy. Nine (37.5\%) patients were weaned to room air in $\leq 7$ days, and another 5 patients were weaned to room air in $\leq 14$ days. The average time for 23 out of the 24 subjects to be weaned to room air was 16.3 days (SD 20.8). The one patient who could not be weaned to room air had C1 AIS A SCI with dysphagia. Speaking valve trials were initiated on $10(41.6 \%)$ patients in $\leq 7$ days and another 9 patients in $\leq 14$ days. On average, the number of days to speaking valve trial from admission was 9.7 days (SD 5.1). All individuals were able to vocalize during their rehabilitation stay using a speaking valve, minimum tracheostomy cuff leak, or total tracheostomy cuff deflation.

Transitioning patients to portable ventilators (enabling them to get up in a wheelchair) occurred in $16(69.6 \%)$ patients in $\leq 7$ days and another 4 patients in $\leq 14$ days. Twenty-three (96\%) individuals were transitioned from the bedside conventional ventilator to the portable ventilators, allowing the patients mobility in a wheelchair, and one patient was completely weaned directly from the bedside conventional ventilator. Average time to use a portable vent was 7.7 days post admission (SD 5.0). All patients on the portable ventilator were able to leave their rooms and participate in therapy and social events. All patients, except one, 
who had neurological levels of C3 or C4 and no evidence of a phrenic neuropathy $(n=13)$ were successfully weaned from mechanical ventilation. Peak inspiratory pressures (PIP) and plateau pressures on the ventilator were measured with the patient in the supine position in bed and in upright positions with abdominal binders on. With the large tidal volume ventilation, PIPs were rarely over $30 \mathrm{cwp}$. One of the subjects underwent one bronchoscopy after transfer to the SCI specialty unit, but this individual had multiple significant medical complications including diabetes mellitus, asthma, and history of cancer and his BMI was 32.9.

Nine patients with level of injury at C1, C2, and $\mathrm{C} 3$ could not be weaned from the ventilator. One patient with C4 AIS B who needed nighttime ventilation was the same patient mentioned above who had multiple significant medical complications and history of cancer and a BMI of 32.9. In 14 patients who were weaned off the ventilator, the average day to be weaned from the time of admission was 27.6 days (SD 12.9 days). Three individuals with C3 AIS A were ventilator weaned in 24 to 62 days (average 43.67 days). Eight individuals with C4 AIS A were ventilator weaned in 14 to 31 days (average 22.13 days). Two individuals with C4 AIS B were weaned from the ventilator in 19 to 22 days (average 20.5 days). One individual with C4 AIS C was weaned in 37 days. This individual had a significant smoking and alcohol use history, had dysphagia, and was 54 years old. Six subjects were decannulated prior to discharge to home, and the average days to be decannulated after admission was 42.0 days ( $S D$ 16.6 days).

Because dysphagia has been shown to be associated with respiratory complications in this patient population, ${ }^{28-30}$ we also evaluated the outcomes of the individuals who had dysphagia. Ten patients $(42 \%)$ were diagnosed as having dysphagia by a Speech-Language Pathologist either on bedside or videofluoroscopic evaluation. Seven of these individuals (4 patients were C4 AIS A, 2 patients were C4 AIS $\mathrm{B}, 1$ patient was C4 AIS C) were neurologically capable of being weaned from the ventilator, and 3 patients had a C1 AIS A injury and remained ventilator-dependent.
Treated with HVtV, HFPV, and MIE, the respiratory outcomes of these patients with dysphagia were as follows: The average day to speaking valve trial was 10 days, and the average day to transition to the portable ventilator was 9.1 days. For the 7 study patients with dysphagia who were neurologically capable of ventilator weaning, the average day to ventilator weaning trial was 5.7 days. The average time to be weaned from the ventilator 24 hours per day for the $4 \mathrm{C} 4$ AIS A patients with dysphagia was 24.5 days.

\section{Discussion}

Respiratory failure and respiratory complications such as atelectasis, bronchospasm, pulmonary edema, and pneumonia are common complications after cervical SCI. The Consortium for Spinal Cord Medicine has published a clinical practice guideline for health care professionals on respiratory management following SCI, which mentions the use of MIE and HFPV. ${ }^{31}$ The use of HVtV, HFPV, and MIE implemented in our SCI unit by respiratory care practitioners in the prevention and treatment of respiratory complications has demonstrated improved outcomes for those with cervical SCI. ${ }^{16,32,33}$

However, in patients with significant comorbidities, weaning may not occur successfully. For example, one patient (C4 AIS B) who had a premorbid history of obesity, asthma, diabetes, and cancer and who was diagnosed with dysphagia was not able to wean off the ventilator for 24 hours daily but required nighttime ventilation and had one bronchoscopy performed while in the SCI unit. These premorbid factors and the presence of dysphagia may have contributed to this inability to totally wean off the ventilator. The patient's BMI was 32.9 ; the possibility of obstructive sleep apnea premorbidly could have been another contributing factor.

Early diagnosis of dysphagia is important in the respiratory management in patients with cervical SCI. The major complications of dysphagia are aspiration and subsequent pneumonitis or pneumonia. Failure to identify the presence of dysphagia increases the need for supplemental oxygen, longer time on the ventilator, and delays in vocalization and participation in rehabilitation 
therapies. In our study, the patients who were identified with dysphagia had improved respiratory outcomes in comparison with their respiratory status prior to admission to our SCI unit. Days to speaking valve trials, time to transition to the portable ventilator, and initial vent weaning trials for 8 of the patients diagnosed with dysphagia were compared with the patients in the study who did not have dysphagia when treated with $\mathrm{HVtV}$, HFPV, and MIE treatments. Two of the 10 patients diagnosed with dysphagia were over the average days to speaking valve trial and transitioning to the portable ventilator. One of these patients was $\mathrm{Cl}$ AIS A and the other was C4 AIS C, had a history of smoking and alcohol use, and was 54 years of age.

There have been 5 published articles addressing the use of MIE specifically in SCI. Compared to the manual cough assist ("quad cough") technique, MIE provides much higher peak expiratory flow $(7.47 \mathrm{~L} / \mathrm{s}$ vs $3.4 \mathrm{~L} / \mathrm{s}) .{ }^{34}$ It appears that the use of MIE in individuals with SCI may have been increasing since Bach's article was published in 1993; but in a most recent study by Schimdt, ${ }^{35}$ only about $64 \%$ of the acute rehabilitation facility was using the MIE device. Given how well the MIE treatment works on secretion management in individuals with acute cervical SCI, the MIE device should be available at all acute rehabilitation facilities that treat individuals with SCI. In particular, those with a prior history of smoking have been shown to benefit from consistent MIE use. ${ }^{25}$ Although there are several published reports and guidelines supporting the use of MIE in individuals with SCI, there is no evidence-based literature describing or supporting the use of HFPV specifically for SCI. ${ }^{3,21,31,32}$ The limitation of this study is that we cannot specifically describe the effectiveness of just HFPV or MIE in this patient population because a combination of respiratory care interventions ( $\mathrm{HVtV}, \mathrm{HFPV}$, and MIE) was used in almost all of the subjects. The efficacy and safety of the use of $\mathrm{HVtV}$ have been previously reported..$^{18,33}$

Many of the respiratory improvements occurred within 7 days of initiating these respiratory care interventions in this study. The average days to be weaned off the ventilator for the 14 patients who could be weaned was 27.6 days. The average days for the patients who were able to be decannulated was 42 days. Patient outcomes improved overall after admission in comparison to respiratory status prior to admission to the SCI specialty unit. When medically stabilized, these individuals can move forward and participate in their rehabilitation programs.

\section{Conclusions}

Respiratory complications after SCI remain the most common reasons for mortality after SCI. ${ }^{36}$ Recognizing and effectively managing the special respiratory complications of individuals with cervical SCI are crucial. Therefore, aggressive respiratory care interventions to prevent respiratory complications need to begin immediately after injury. Because almost all individuals in this study received all 3 interventions $(\mathrm{HVtV}, \mathrm{HFPV}$, and MIE), it is difficult to identify whether any one particular intervention is more effective than the other. A combination of these 3 interventions seems indicated for individuals after cervical SCI who are at risk for respiratory failure.

\section{Acknowledgments}

Previous publication: Preliminary findings on this project have been presented as a symposium presentation at the 2012 ASIA conference.

Funding support: This study was partially funded by the US Department of Education, Office of Special Education and Rehabilitative Services, National Institute on Disability and Rehabilitation Research, grant H133G080165.

Additional contributions: We thank John A. Dietrich, RRT, RCP, NPS, Paul Barraza, RRT, RCP, and Craig Ivie, RCP, NPS, of Santa Clara Valley Medical Center Respiratory Care Services Department for their respiratory care technical assistance. We also acknowledge editorial assistance by James Chang, BA, of Santa Clara Valley Medical Center Rehabilitation Research Center. 
1. Jackson, $A B$, Groomers TE. Incidence of respiratory complications following SCI. Arch Phys Med Rehabil. 1994;75:270-275.

2. Lemons VR, Wagner FC Jr. Respiratory complications after cervical spinal cord injury. Spine. 1994;19:23152320.

3. Berlly M, Shem K. Respiratory management during the first five days after spinal cord injury. J Spinal Cord Med. 2007;30:309-318.

4. McMichan JC, Michel L, Westbrook PR. Pulmonary dysfunction following traumatic quadriplegia. Recognition, prevention, and treatment. JAMA. 1980;243(6):528-531.

5. Brown R, DiMarco AF, Hoit JD, Garshick E. Respiratory dysfunction and management in spinal cord injury. Respir Care. 2006;51(8):853-868; discussion 869870.

6. Bhaskar KR, Brown R, O'Sullivan DD, Melia S, Duggan $M$, Reid L. Bronchial mucus hypersecretion in acute quadriplegia. Macromolecular yields and glycoconjugate composition. Am Rev Respir Dis. 1991;143:640-648.

7. Slack RS, Shucart W. Respiratory dysfunction associated with traumatic injury to the central nervous system. Clin Chest Med. 1994;15(4):739-749.

8. Carter RE, Donovan WH, Halstead L, Wilkerson MA. Comparative study of electrophrenic nerve stimulation and mechanical ventilatory support in traumatic spinal cord injury. Paraplegia. 1987;25(2):86-91.

9. Lanig IS, Peterson WP. The respiratory system in spinal cord injury. Phys Med Rehabil Clinics of North Am. 2000;11:29-43.

10. Claxton R, Wong D, Chung R, Fehlings M. Predictors of hospital mortality and mechanical ventilation in patients with cervical spinal cord injury. Can J Anaesth. 1998;45:144-149.

11. Tromans AM, Mecci M, Barrett FH, Ward TA, Grundy DJ. The use of the BiPAP system in acute spinal cord injury. Spinal Cord. 1998;36:481-484.

12. Bach JR. Noninvasive alternatives to tracheostomy for managing respiratory muscle dysfunction in spinal cord injury. Top Spinal Cord Inj Rehabil. 1997;2:49-58.

13. Van Houtte S, Vanlandewijck Y, Gosselink R. Respiratory muscle training in persons with spinal cord injury: a systematic review. Respir Med. 2006;100(1 1):1886-1895.

14. Pilbeam S, Cairo JM. Mechanical ventilation. In: Hutchinson M, Boyle MK, Lastarria $M$, eds. Physiological and Clinical Applications. 4th ed. St. Louis, MO: Mosby Elsevier; 2006.

15. Acute Respiratory Distress Syndrome Network. Ventilation with lower tidal volumes as compared with traditional tidal volumes for acute lung injury and the acute respiratory distress syndrome. $N$ Engl J Med. 2000;342:1301-1308.

16. Peterson P, Brooks CA, Mellick D, Whiteneck G. Protocol for ventilator management in high tetraplegia. Top Spinal Cord Inj Rehabil. 1997:2:101-106.
17. Boynton JH Jr, Hawkins K, Tansey K. Small or large tidal volumes: the dilemma of spinal cord injury. Respir Care J. 2005; 11:1522.

18. Lammertse $D$, Fenton J, Werner $M$, et al. High versus standard tidal volumes in ventilator weaning for persons with subacute cervical spinal cord injury. Top Spinal Cord Inj Rehabil. 2012;18(1):213.

19. Massaro GD, Massaro D. Morphologic evidence that large inflations of the lung stimulate secretion of surfactant. Am Rev Respir Dis. 1983;127(2):235-236.

20. Haas CF, Loik PS, Gay SE. Airway clearance applications in the elderly and in patients with neurologic or neuromuscular compromise. Respir Care. 2007;52(10):1362-1381.

21. Chatburn RL, Deem S. Respiratory controversies in the critical care setting. Should weaning protocols be used with all patients who receive mechanical ventilation? Respir Care. 2007;52(5):609-619.

22. Bach JR. Inappropriate weaning and late onset ventilatory failure of individuals with traumatic spinal cord injury. Paraplegia. 1993;31(7):430-438.

23. Homnick DN. Mechanical insufflation-exsufflation for airway mucus clearance. Respir Care. 2007;52(10):12961305; discussion 1306-1307. Erratum in: Respir Care. $2011 ; 56(6): 888$.

24. Pillastrini P, Bordini S, Bazzocchi G, Belloni G, Menarini M. Study of the effectiveness of bronchial clearance in subjects with upper spinal cord injuries: examination of a rehabilitation program involving mechanical insufflation and exsufflation. Spinal Cord. 2006;44(10):614-616.

25. Crew JD, Svircev JN, Burns SP. Mechanical insufflation-exsufflation device prescription for outpatients with tetraplegia. J Spinal Cord Med. 2010;33(2): 128-134.

26. Suri P, Burns SP, Bach JR. Pneumothorax associated with mechanical insufflation-exsufflation and related factors. Am J Phys Med Rehabil. 2008; 87(11):951-955.

27. Chatwin $M$, Simonds AK. The addition of mechanical insufflation/exsufflation shortens airway-clearance sessions in neuromuscular patients with chest infection. Respir Care. 2009;54(11):1473-1479.

28. Shem K, Castillo K, Naran B. Factors associated with dysphagia in individuals with high tetraplegia. Top Spinal Cord Inj Rehabil. 2005;10(3):8-18.

29. Shem K, Castillo K, Wong SL, Chang J, KolakowskyHayner S. Dysphagia and respiratory care in individuals with tetraplegia: incidence, associated factors, and preventable complications. Top Spinal Cord Inj Rehabil. 2012;18(1):15-22.

30. Shem K, Castillo K, Wong S, Chang J. Dysphagia in individuals with tetraplegia: incidence and risk factors. J Spinal Cord Med. 2011;34(1):85-92.

31. Consortium for Spinal Cord Medicine. Respiratory management following spinal cord injury: a clinical practice guideline for health-care professionals. J Spinal Cord Med. 2005;28:259-293. 
32. Wallbom A, Naran B, Thomas E. Acute ventilator management and weaning in individuals with high tetraplegia. Topics Spinal Cord Inj Rehabil. 2005; 10:3:1-7.

33. Peterson WP, Barbalata L, Brooks CA, Gerhart KA, Mellick DC, Whiteneck GG. The effect of tidal volumes on the time to wean persons with high tetraplegia from ventilators. Spinal Cord. 1999;37:284-288.

34. Bach JR. Mechanical insufflation-exsufflation. Comparison of peak expiratory flows with manually assisted and unassisted coughing techniques. Chest. 1993;104(5): 1553-1562.

35. Schmidt JK, Stiens S, Trincher R, Lam M, Aarkarati $M$, Linder $S$, Ho $C$. Survey of use of the insufflatorexsufflator in patients with spinal cord injury. J Spinal Cord Med. 2007;30(2);127-130.

36. DeVivo MJ, Chen Yuing, Kruase J, Saunders LL. Trends in age-adjusted cause-specific mortality rates after spinal cord injury. Top Spinal Cord Inj Rehabil. 2012;18(1):214 